\title{
Vigilância epidemiológica de tuberculose em portadores de HIV na região norte, no
}

\section{período de 2011 a 2020}

\author{
Epidemiological surveillance of tuberculosis in HIV carriers in the northern region, from 2011 to
}

2020

Vigilancia epidemiológica de la tuberculosis en portadores de VIH en la región norte, de 2011 a

Tayla Souza dos Santos ORCID: https://orcid.org/0000-0002-8855-4483 Centro Universitário da Amazônia, Brasil E-mail: taylasouza75@gmail.com

Elinelson Sousa da Silva Junior ORCID: https://orcid.org/0000-0003-2709-0002 Centro Universitário da Amazônia, Brasil E-mail: elinelsonjunior01@gmail.com

Maria da Conceição Caetano de Siqueira ORCID: https://orcid.org/0000-0003-1731-4076 Centro Universitário da Amazônia, Brasil E-mail: siqueira_44@hotmail.com

\begin{abstract}
Resumo
Introdução: a tuberculose (TB) é uma doença infectocontagiosa grave, que se apresenta como um problema de saúde pública no mundo, principalmente nos países subdesenvolvidos. A coinfecção TB/HIV, demostram-se como um desafio aos serviços de saúde pública, devido às proeminentes taxas de abandono do tratamento e afigura-se como uma das grandes causas de mortes e de atendimento hospitalar no Brasil, São Paulo foi um dos principais estados com o alto número de pessoas acometidas com Tuberculose e HIV. Objetivo: abordar o perfil epidemiológico dos pacientes diagnosticados com tuberculose que são portadores de HIV analisando as variáveis de incidência, mortalidade e abandono do tratamento no período de 2011 a 2020. Metodologia: Trata -se de um estudo descritivo de caráter quantitativo, onde foi realizado uma revisão epidemiológica comparativa, correlacionando os dados anuais de cunho público coletados no Sistema de Informação de Agravos de Notificação (SINAN). Resultados e discussão: O estado do Amazonas é a unidade da federação com a maior incidência de TB no Brasil, com 68,4 casos novos por 100 mil habitantes. A taxa de mortalidade no brasil sofreu uma redução, embora ainda alarmante, cerca de 6.000 óbitos são notificados todo ano, sendo sua maior incidência estados com as maiores taxas de mortalidade nos estados do Amazonas e Pará, seguido de Rondônia, Roraima e Tocantins mantiveram sua posição. os números de casos por abandono de tratamento foram significativos, principalmente no AM, PA e RO. Conclusão: Foi possível observar a importância da vigilância epidemiológica de tuberculose em portadores de HIV na Região Norte. Observou - se uma relação entre os níveis mais baixos de escolaridade com a desistência do tratamento.
\end{abstract}

Palavras-chave: Tuberculose; HIV; Epidemiologia.

\begin{abstract}
Introduction: tuberculosis (TB) is a serious infectious disease, which presents itself as a public health problem in the world, especially in underdeveloped countries. The TB/HIV co-infection, demonstrated as a challenge to public health services, due to the prominent rates of treatment abandonment and appears as one of the major causes of death and hospital care in Brazil, São Paulo was one of the main states with the high number of people afflicted with Tuberculosis and HIV. Objective: to address the epidemiological profile of patients diagnosed with tuberculosis who are HIV carriers, analyzing the variables of incidence, mortality and treatment dropout in the period from 2011 to 2020. Methodology: This is a descriptive study of a quantitative nature, where it was carried out a comparative epidemiological review, correlating the annual public data collected in the Notifiable Diseases Information System (SINAN). Results and discussion: The state of Amazonas is the federation unit with the highest incidence of TB in Brazil, with 68.4 new cases per 100,000 inhabitants. The mortality rate in Brazil has suffered a reduction, although still alarming, about 6,000 deaths are reported every year, with the highest incidence being states with the highest mortality rates in the states of Amazonas and Pará, followed by Rondônia, Roraima and Tocantins maintained their position. the numbers of cases due to treatment dropout were significant, mainly in AM, PA and RO. Conclusion: It
\end{abstract}


was possible to observe the importance of epidemiological surveillance of tuberculosis in HIV carriers in the North Region. A relationship between lower levels of education and treatment dropout was observed.

Keywords: Tuberculosis; HIV; Epidemiology.

\begin{abstract}
Resumen
Introducción: la tuberculosis (TB) es una enfermedad infecciosa grave que se presenta como un problema de salud pública en el mundo, especialmente en los países subdesarrollados. La coinfección TB / VIH, demostrada como un desafío para los servicios de salud pública, debido a las destacadas tasas de abandono del tratamiento y aparece como una de las principales causas de muerte y atención hospitalaria en Brasil, São Paulo fue uno de los principales estados con el elevado número de personas afectadas por la tuberculosis y el VIH. Objetivo: abordar el perfil epidemiológico de los pacientes diagnosticados de tuberculosis portadores de VIH, analizando las variables de incidencia, mortalidad y abandono del tratamiento en el período 2011 a 2020. Metodología: Se trata de un estudio descriptivo de carácter cuantitativo, donde se realizó una revisión epidemiológica comparativa, correlacionando los datos públicos anuales recolectados en el Sistema de Información de Enfermedades Notificables (SINAN). Resultados y discusión: El estado de Amazonas es la unidad federativa con mayor incidencia de TB en Brasil, con 68,4 casos nuevos por 100.000 habitantes. La tasa de mortalidad en Brasil ha sufrido una reducción, aunque sigue siendo alarmante, se reportan alrededor de 6.000 muertes cada año, siendo la mayor incidencia los estados con mayores tasas de mortalidad en los estados de Amazonas y Pará, seguidos de Rondônia, Roraima y Tocantins mantuvieron su posición. el número de casos por abandono del tratamiento fue significativo, principalmente en AM, PA y RO. Conclusión: se pudo observar la importancia de la vigilancia epidemiológica de la tuberculosis en portadores de VIH en la Región Norte. Se observó una relación entre niveles más bajos de educación y abandono del tratamiento.
\end{abstract}

Palabras clave: Tuberculosis; VIH; Epidemiología.

\title{
1. Introdução
}

A tuberculose (TB) é uma doença infectocontagiosa grave, que se apresenta como um problema de saúde pública no mundo, principalmente nos países subdesenvolvidos. A doença é transmissível e afeta prioritariamente os pulmões, podendo ainda infectar outros órgãos, é causada pelo Mycobacterium tuberculosis ou bacilo de Koch. A forma pulmonar é a única responsável pela transmissão da doença, sendo a mais frequente e relevante para a saúde pública quando positivada à baciloscopia. A doença afeta a população de modo geral sendo mais frequente em indivíduos com baixa residência. De acordo com West (2017), a incidência de coinfecção pelo Mycobacterium tuberculosis em pacientes positivo para HIV ainda é um dos principais problemas de saúde pública tanto em âmbito nacional como em outros países.

A coinfecção TB/HIV, demostram-se como um desafio aos serviços de saúde pública, devido às proeminentes taxas de abandono do tratamento e afigura-se como uma das grandes causas de mortes e de atendimento hospitalar no Brasil, São Paulo foi um dos principais estados com o alto número de pessoas acometidas com Tuberculose e HIV, demostrando quase $10 \%$ dos casos de coinfecção de TB/HIV (Sena, 2020).

A TB é uma das 10 principais causas de morte no mundo e a maior causa de mortalidade entre as doenças infecciosas. Em todo o globo são estimados anualmente 10 milhões de novos casos e 1,57 milhão de mortes pela doença. Entre as pessoas com infecção pelo HIV, uma média 900 mil novos diagnósticos e 300 mil óbitos são registrados a cada ano. O Brasil encontrase entre os 22 países que, juntos, concentram $80 \%$ dos casos de TB no mundo. No país, são notificados cerca de 67 mil casos novos e ocorrem 4,5 mil mortes anuais em decorrência de TB (Junior, 2019).

O surgimento do HIV agravou um elevado número de casos de tuberculose (TB) em todo o mundo. A doença afeta os portadores de HIV em qualquer fase da AIDS, sendo a principal causa de morte entre estes. No Brasil, a incidência da coinfecção TB/HIV cresceu vertiginosamente entre os anos de 2010 a 2018. Em 2019, foram notificados 91.056 casos novos de Tuberculose, desses são também portadores de HIV/AIDS 4.230 pessoas. Dentre as pessoas com coinfecção TB-HIV no Brasil, em 2019, apenas 47,5\% realizaram terapia antirretroviral (TARV) durante o tratamento da TB (Souza, et. al. 2020). O autor ressalta que pessoas que possuem a infecção HIV/AIDS tem 25 vezes maior risco de adoecimento por tuberculose e 
consiste em uma das condições de maior impacto na morbimortalidade pois leva milhares de pessoas a óbitos no Brasil e no mundo.

Mundialmente, estima-se 9,4 milhões de pessoas com tuberculose ativa a cada ano, 1,4 milhões são portadores do HIV (Silva et al., 2017). É amplamente aceito que o HIV causa uma depleção de células T CD4+ e esta contribui para a susceptibilidade dos coinfectados ao Mycobacterium tuberculosis, bem como este subconjunto de células T é importante para o controle da TB. No entanto, o HIV tem efeitos sobre outras células, incluindo macrófagos, influencia a produção de citocinas que também podem impedir que um indivíduo com infecção de TB inicial ou latente evolua para TB ativa (Diedrich et al., 2010).

É uma doença de alta transmissibilidade, e tem maior probabilidade de ser desenvolvida em indivíduos positivos para HIV devido a imunossupressão do organismo do portador de HIV, A partir de tal constatação levantou-se o interesse pelo tema pesquisado, pela alta incidência de tuberculose/HIV, na carência de informações das Pessoas Vivendo com o HIV, e como bruscamente os níveis sociodemográfico elevando na região do norte, portanto, é fundamental um aprimoramento de uma integração de serviços e a necessidade de realizar uma análise sobrea a importância da vigilância epidemiológica sobre esses indivíduos com tuberculose, mas que são portadores de HIV positivo.

A tuberculose (TB) é uma enfermidade que data milhões de anos e tem persistido na sua proliferação até nos dias de hoje. A TB é responsável por altíssimos índices de mortalidade pela falta de prevenção e cuidados adequados, representando assim, um grave problema de saúde pública no Brasil.

A Organização Mundial da Saúde (OMS) anunciou que em 2002 os casos de TB apontaram altíssimos índices de doentes, entretanto, a OMS ainda aponta que 1/3 da população mundial, ainda hoje, está infectado pelo Mycobacterium tuberculosis. Em 1993, a Organização Mundial de Saúde também foi enfática em considerar a TB como situação de emergência sanitária, haja vista que 8 milhões de pessoas adoecem por ano e a doença ainda leva à óbito cerca 2,9 milhões destas. Os países subdesenvolvidos e em desenvolvimento que apresentam condições de pobreza são os mais afetados, registrando em 95\% dos casos uma taxa bruta de 8 milhões de casos anuais, incluindo o Brasil.

Considera-se ainda que a TB possui relação intrínsecas com as condições de saneamento básico e também com a classe social do indivíduo. Pessoas que vivem em maior situação de miséria são as mais expostas à manifestação do bacilo. No Brasil, têm nas suas ocorrências este histórico, ou seja, os casos da doença são notificados principalmente nas regiões de periferia ou em áreas de aglomeração ou comunidades, antes chamadas de favelas.

Seguindo este mapa de intempéries, destaca-se ainda que além da situação de moradia, a alimentação se torna um fator preponderante para a disseminação da infecção, associada também com a ingestão de álcool, tabaco e outras drogas (Campos, 2006).

Para Smeltzer e Bare (1999), esta patologia tem características infecciosas ocasionada pelo Mycobacterium tuberculosis transmitido por via aérea, a partir de um indivíduo infectado para o sadio. Quando ocorre a transmissão da TB, o bacilo pode se instalar em diversos órgãos, quer seja durante a primo-infecção, que corresponde aos casos de imunidade específica ainda não desenvolvida, ou mesmo em casos de diminuição na capacidade do hospedeiro em manter o bacilo em seus sítios de implantação, ou seja, nos casos em que o sistema imunológico do hospedeiro não consegue eliminar o bacilo, tornando-se suscetível à ação da doença. Vale ressaltar, ainda, que uma vez o indivíduo sendo infectado, a manifestação da TB pode ocorrer em qualquer momento da vida (Campos, 2006).

De acordo com Hayne (2018), a interação entre o HIV e o Mycobacterium Tuberculosis é complexa. Ambos agem sobre os elementos do sistema imune e afetam-se mutuamente. O HIV apresenta maior afinidade por células com alta quantidade de receptores CD4+, em contrapartida, o bacilo de Koch possui predisposição pelas vias aéreas e sua infecção só ocorre quando o mesmo adentra os alvéolos pulmonares, atingindo os linfonodos. 
Segundo o Brasil (2019), salienta que as pessoas, vivendo com HIV (PVHIV) estão mais propensas a desenvolver tuberculose (TB) quando comparadas à população geral. Em 2016, no mundo, foram notificados 476.774 casos de coinfecção TB-HIV (13\% do total de casos de TB notificados), dos quais $85 \%$ estavam em TARV. Embora tenha havido um aumento das notificações de TB associada ao HIV nos últimos anos, em 2016, elas corresponderam apenas a $46 \%$ do total estimado pela OMS, patenteando as lacunas ainda existentes na detecção, tratamento e acompanhamento apropriado dessas pessoas.

Segundo a Resende (2017, p. 23),

O tratamento da coinfecção TB e HIV/aids envolve dois esquemas: o tratamento da TB e o da infecção pelo HIV/aids. Para os novos casos de TB o tratamento se baseia na combinação de quatro fármacos, sendo Rifampicina (R), Isoniazida (H), Pirazinamida (Z) e Etambutol (E) na fase intensiva, de duração de dois meses. Os fármacos R e H são utilizados por quatro meses na fase de manutenção. Em casos especiais o tratamento pode ser superior a seis meses. A apresentação desse esquema é em dose fixa combinada dos quatro fármacos (RHZE). Em tratamentos especiais, como hepatopatias, reações adversas maiores, HIV/aids e uso de imunossupressores é indicado o uso de esquemas especiais.

Como o tratamento de tuberculose requer um tratamento especifico, em questão de esquema de medicamento se torna difícil, sendo que o mais difícil ainda quando o cliente e o portador do vírus do HIV, no qual alguns remédios de tratamento de TB, não fazem efeitos por motivos de interações medicamentosas, com isso a maioria dos clientes acabam desistindo do tratamento, trazendo e desenvolvendo complicações graves e até evoluindo para um óbito, nos casos de clientes que requer continuação do tratamento, mesmo após ter desistido é desenvolvido um novo esquema de tratamento em questão de remédios, por motivos que o Mycobacterium tuberculosis já se torna imune a certos antibióticos por não faz.

O presente artigo tem como objetivo abordar o perfil epidemiológico dos pacientes diagnosticados com tuberculose que são portadores de HIV analisando as variáveis de incidência, mortalidade e abandono do tratamento no período de 2011 a 2020.

\section{Metodologia}

Trata -se de um estudo descritivo de caráter quantitativo, onde foi realizado uma revisão epidemiológica comparativa, correlacionando os dados anuais de cunho público coletados no Sistema de Informação de Agravos de Notificação (SINAN).

Para atribuição da característica quantitativa, levou -se em consideração o que foi proposto por Aragão (2011) uma vez que os dados são comparados em gráficos ressaltando sua estatística e generalizando a ação do coletivo. Gerhardt e Silveira (2009), corroboram que o estudo descritivo é uma das classificações das pesquisas científicas, cujo objetivo é descrever as características de uma população, um fenômeno ou experiência para o estudo completo.

Foi incluído neste estudo o número total de casos confirmados de tuberculose em portadores de HIV na Região Norte no Brasil, referentes aos períodos de 2011 a 2020; dados de casos confirmados de tuberculose em portadores de HIV. Tendo como critério de exclusão, aqueles cuja variável HIV foi preenchida como não realizada, negativa, em andamento, ou em branco.

As variáveis estudadas para os casos confirmados de tuberculose em portadores de HIV foram: unidade da Federação de notificação; ano de diagnóstico; casos confirmados por região; região de notificação. Sendo utilizado os seguintes descritores: taxa de incidência; taxa de alta por cura; taxa de mortalidade e taxa de abandono por tratamento.

As informações sobre os casos confirmados de tuberculose em portadores de HIV e a população utilizada para o cálculo da incidência (100.000 habitantes) foram obtidos no site do SINAN, disponibilizados na plataforma do DATASUS. 
O valor do percentual de tuberculose em portadores de HIV nessa média de tempo foi obtido entre a quociente da soma dos números notificados de casos novos de tuberculose em todas as formas no mesmo período, por unidade da federação de notificação, de acordo com a formula a seguir:

Casos confirmados de tuberculose em portadores de HIV=

Número de casos novos de tuberculose/HIV x 100.000

Número de habitantes população por estados

Os dados e valores obtidos da tuberculose em portadores de HIV por estados, foram reproduzidos em gráficos de distribuição espacial da TB em portadores de HIV na Região Norte. As análises e os gráficos foram feitos por meio dos softwares TABNET, Microsoft Excel 2016, Mistério da Saúde, Scielo e Revistas de cunho científico.

Os dados foram obtidos dos bancos de dados do SINAN pelo TABNET, disponibilizado pelo DATASUS, razão pela qual o estudo não foi submetido a um Comitê de Ética em Pesquisa, mas foram sondados todos os conteúdos respeitando os princípios éticos estabelecidos na resolução 466/12.

\section{Resultados}

No período entre 2011 a 2020, foram notificados 10.070 casos novos de tuberculose em portadores de HIV na região norte. A situação de vigilância epidemiológica é expressada nos gráficos a seguidamente. O Gráfico 1 apresenta a distribuição da situação anual dos casos confirmados de tuberculose em portadores de HIV na Região Norte no período avaliado.

Gráfico 1. Casos confirmados de tuberculose em portadores de HIV na região norte do Brasil no período de 2011 a 2020.

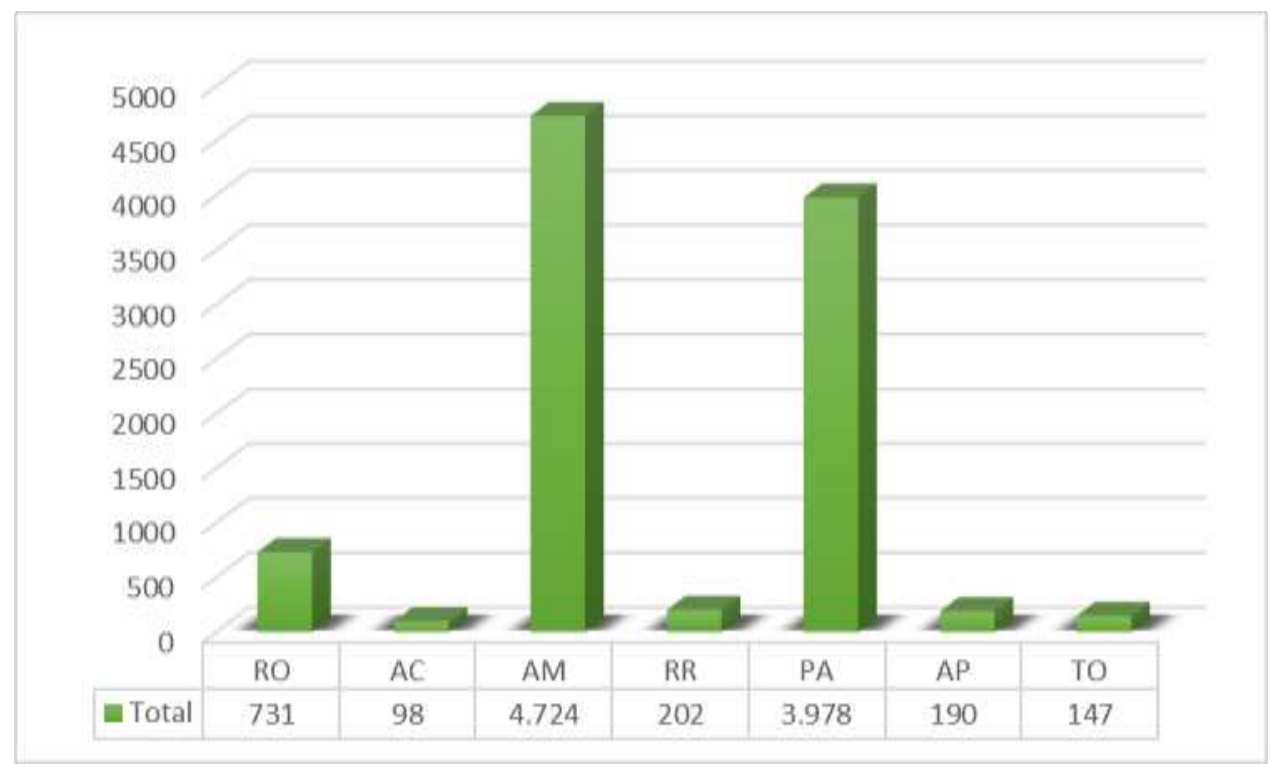

Fonte: Santos et al., (2021).

O Gráfico 1 demostrou um crescimento vertiginosamente de tuberculose em portadores de HIV nos estados do Amazonas (4.724), Pará (3.978) e Rondônia (731), já o Acre (98) e Tocantins (147) tornando-se como os estados com menores ocorrência de casos confirmados no decorrer do período avaliado de 2011 a 2020. 
Observa-se que no Gráfico 2, a taxa de incidência de tuberculose em portadores HIV na Região Norte, sendo notável que ocorreu uma oscilação no decorre de 10 anos destacando-se os estados do AM que chegou a 120,24 por 100.000 habitantes, PA com 47,82 por 100.000 habitantes e RO com 42,86 por 100.000 habitantes, sendo considerados os estados com as maiores taxa de incidência, já no AC chegou 11,81 por 100.000 habitantes e TO com 9,67 por 100.000 habitantes, sendo considerados os estados que tiveram as menores taxa de casos novos de tuberculose em portadores de HIV nesse período de tempo.

Gráfico 2. Taxa de incidência de tuberculose em portadores de HIV no Brasil na região norte, no período de 2011 a 2020.

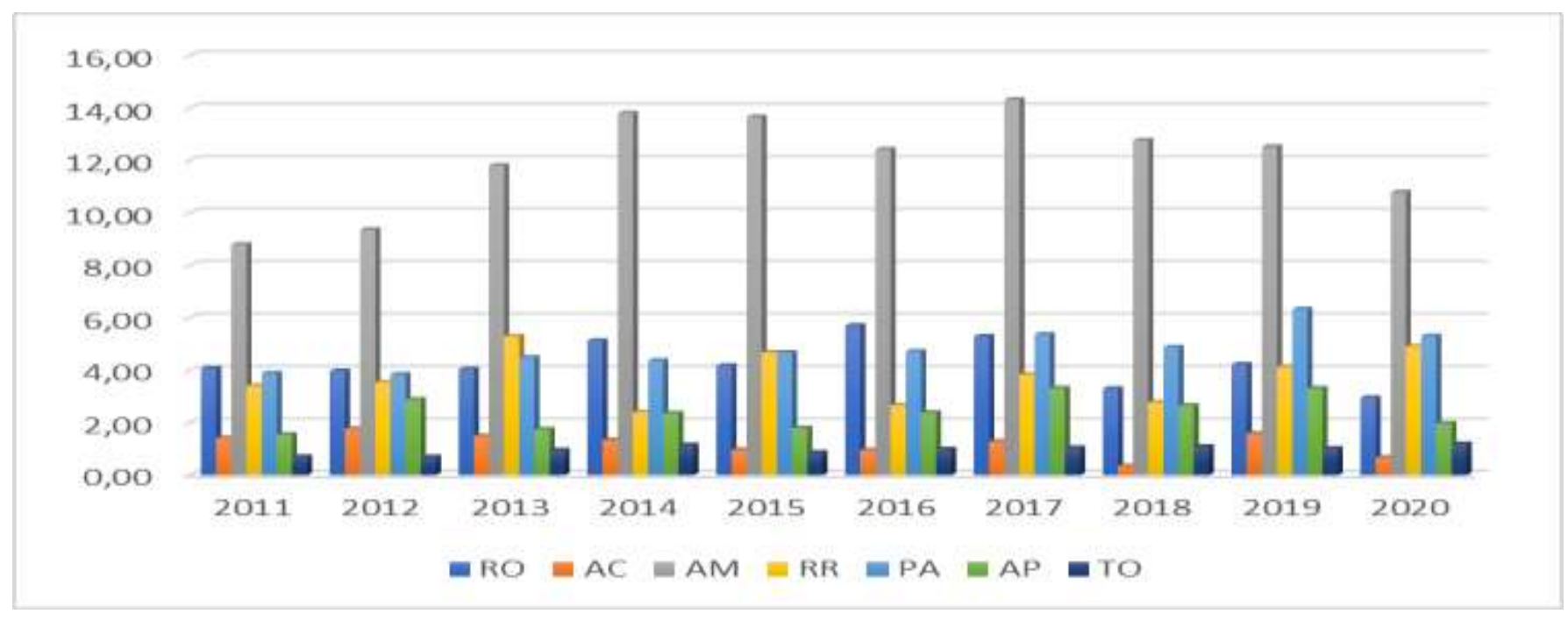

Fonte: Santos et al., (2021).

Em relação ao critério de casos confirmados de cura no Brasil, nota-se que no período estudado, obteve-se confirmações com os maiores números de casos nos estados do Amazonas (2.392) casos de cura, em segundo lugar o estado do Pará (1.884) e o terceiro o estado de Rondônia (336) e com os números menores de notificações foram os estados do Acre (63) e Tocantins (56), conforme observado no Gráfico 3.

Gráfico 3. Casos confirmados de cura de tuberculose em portadores de HIV no brasil na região norte no período 2011 a 2020.

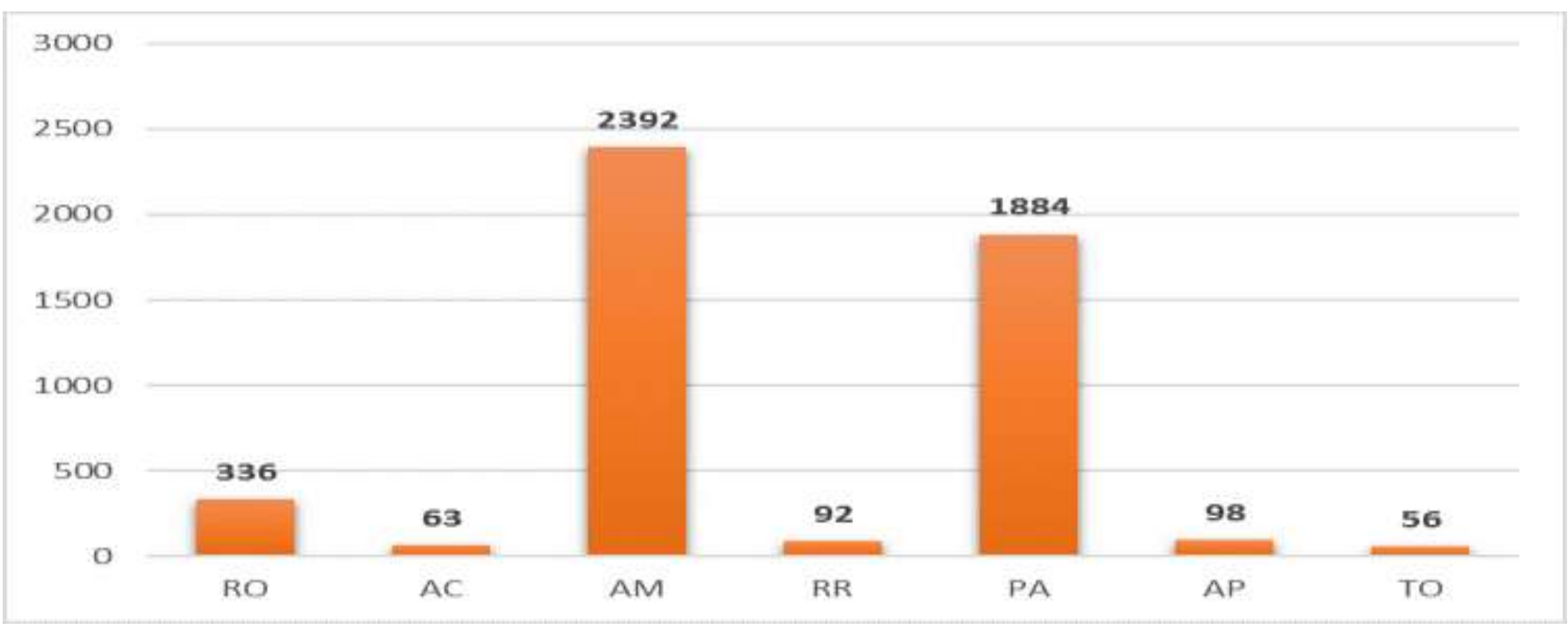

Fonte: Ministério da Saúde/SVS - Sistema de Informação de Agravos de Notificação - Sinan Net. 
Avaliados os dados relacionados acima no gráfico, notou-se que os índices de mortalidade nos períodos estabelecidos mantiveram-se, sendo os estados mais afetados foram o AM (177), PA (129) e RO (13) casos no quais foram identificados, já o RR (3) e TO (3) mantiveram-se os mesmos níveis de mortalidade no período de 2011 e 2020, conforme observado no Gráfico 4.

Gráfico 4. Casos confirmados de mortalidade na região norte no Brasil no período de 2011 a 2020.

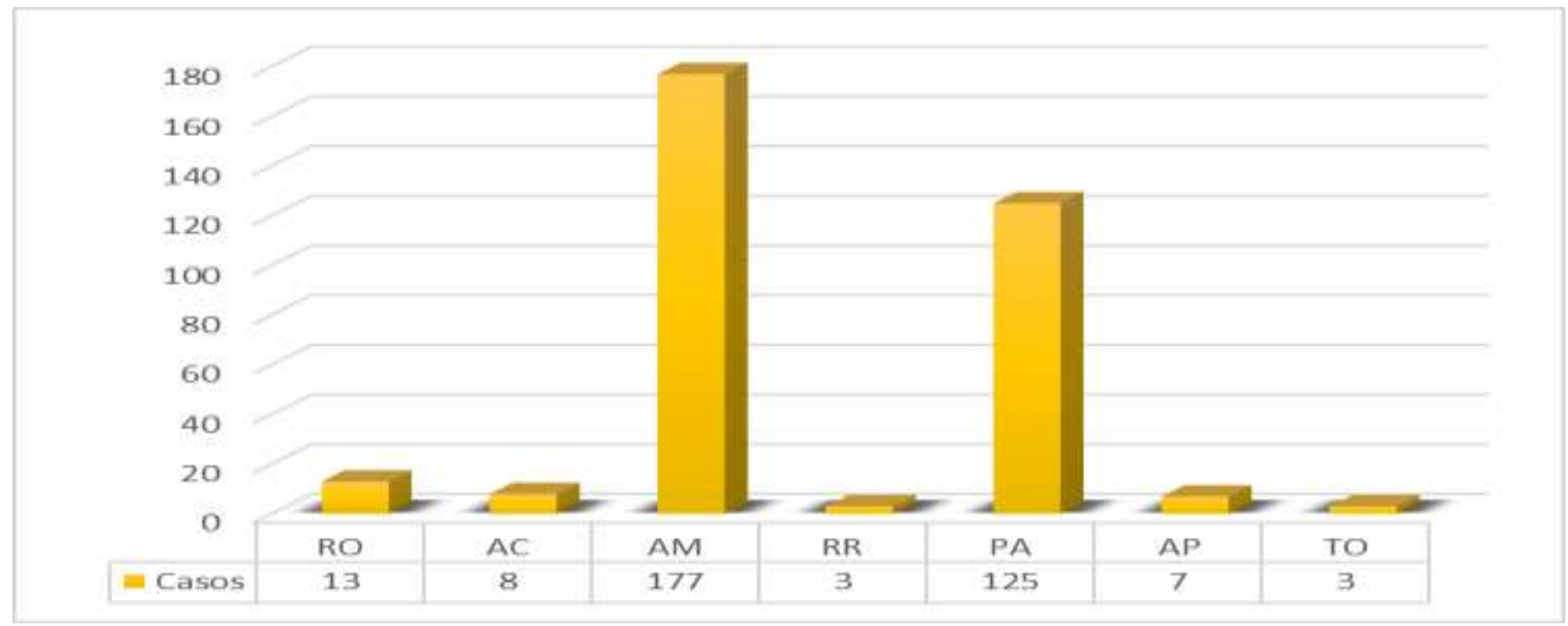

Fonte: Santos et al., (2021).

No Gráfico 5, foram possíveis observa que os números de casos por abandono de tratamento foram significativamente enormes, principalmente no AM (766), PA (537) e RO (171) por casos confirmados de abandono de tratamento, o AC (2) e RR (20) apresentou-se como os estados com menores casos de abandonos, sendo notável o destaque dos mesmos estados citadas anteriormente.

Gráfico 5. Casos confirmados de abandono de tratamento na região norte no Brasil no período de 2011 a 2020.

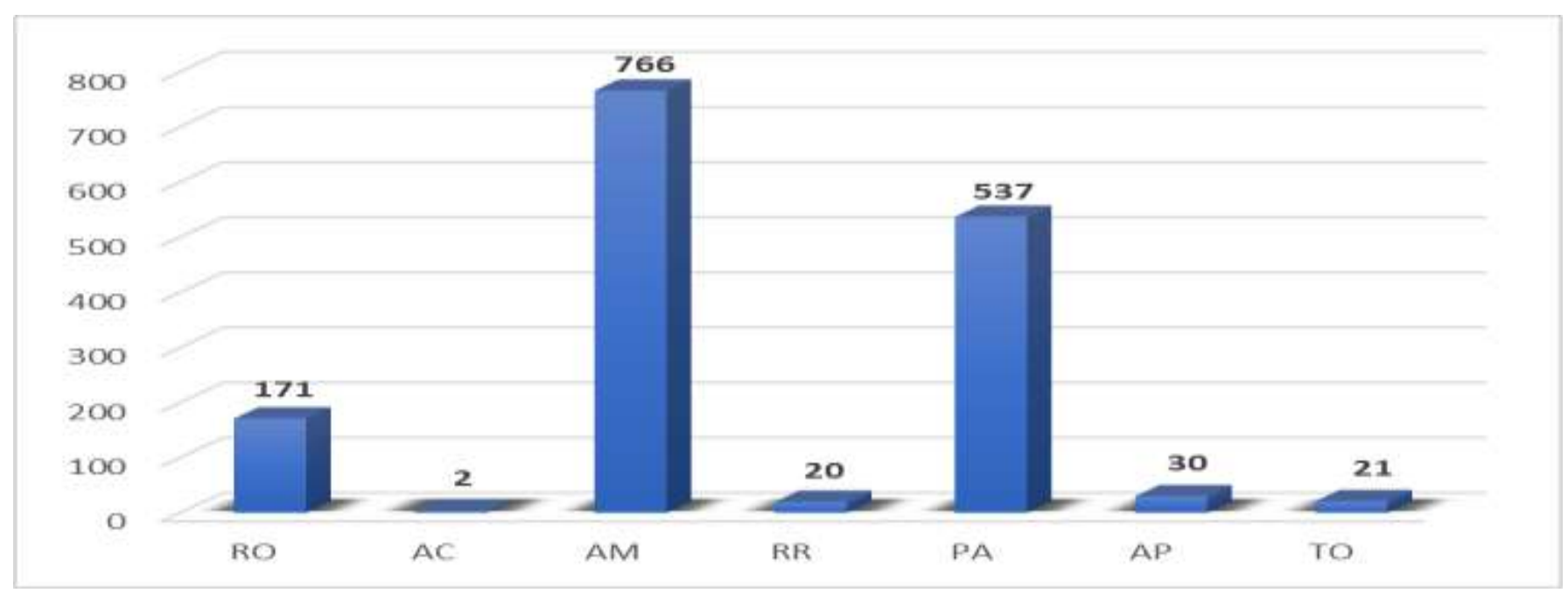

Fonte: Santos et al., (2021). 


\section{Discussão}

De acordo com o Boletim Epidemiológico de Tuberculose do Brasil (2021), o Brasil continua entre os 30 países de alta carga para a TB e para coinfecção TB-HIV, sendo, portanto, considerado prioritário para o controle da doença no mundo pela Organização Mundial de Saúde (OMS).

Segundo o OMS (2021), enfatizar que a pandemia de COVID-19 contribuiu para crescimento e reverteu anos de progresso global no combate à tuberculose e, pela primeira vez em mais de uma década, as mortes pela doença aumentaram, de acordo com o relatório global da Organização Mundial da Saúde. Em 2020, mais pessoas morreram de tuberculose, porém houve uma redução no número de notificação e consequentemente a diminuição de pessoas recebendo tratamento preventivo relacionado ao ano de 2019, já que as pessoas ficaram temerosas em comparecer as unidades de saúde para realizar consultas, sendo que os gastos gerais com serviços essenciais para a doença diminuíram.

Notou -se no estudo de Barreto et al. (2020), que os casos confirmados de tuberculose são menores comparado a Região Norte no período de 5 anos, sendo que houve a notificação de 4.914 casos novos de TB, tendo uma média de $16,67 \%$ casos por ano. Sendo que em 2011 foi o ano com o maior quantitativo apresentando 18,68\% casos, e em 2015 o ano com menor representatividade, dispondo de $13,90 \%$ casos na Região do Nordeste. (Gráfico 1)

Para Oliveira et. al. (2020), comparado ao estudo, enfatiza que a taxa média de incidência no ano de 2008 foi de 47,86/100.000 habitantes da Região Nordeste. Em 2009, aumentou para 48,29/100.000 habitantes, sendo que durante esse ano houve a maior média durante todo o período do estudo. Nos anos seguintes, ocorreram oscilações nos valores da taxa de notificação: 2010 apresentou uma diminuição, sucedido por ligeiro aumento em 2011. Logo em seguida, houve um período de notória redução no número de casos entre os anos de 2012 a 2015 (de 45,19/100.000 habitantes para 39,27/100.000 habitantes).

Em 2015 foi constatada a menor taxa média durante o período estudado, ou seja, 39,27/100.000 habitantes. Posteriormente, houve um breve aumento em 2016, representado pela taxa média de 39,39/100.000 habitantes. Os anos de 2017 e 2018 seguiram o índice de crescimento iniciado no ano de 2016, com 41,59/100.000 habitantes e 43,35/100.000 habitantes respectivamente.

Assim, de acordo com o que mostra o gráfico 2, o Estado do Amazonas é a unidade da federação com a maior incidência de TB no Brasil, com 68,4 casos novos por 100 mil habitantes. As pessoas que convivem com o vírus HIV, tem 28 vezes mais chances de contrair a tuberculose, do que as que não portam o vírus, no entanto, isto é um dado alarmante e preocupante, pois a soropositividade para HIV, vulnerabiliza e deixa o organismo suscetível a infecções, podendo chegar entre elas a tuberculose, dentro de qualquer uma das fases da soropositividade. Sabemos que a tuberculose é uma doença oportunista e pode ser transmitidas a outras pessoas.

Robbins et al., (2005), enfatizam que após a exposição inicial ao Mycobacterium Tuberculosis ocorre uma resposta imunológica no hospedeiro, o qual adquire resistência e hipersensibilidade, com suscetibilidade para a resposta inflamatória iniciar assim, a doença passa a se desenvolver na primeira exposição ou em exposições futuras, podendo ocorrer evolução para suas formas mais graves.

A vigilância eficaz epidemiológica e o trabalho de conscientização são elementos importantes para seu controle, prioritariamente avaliando o panorama de cada estado, a exemplo de Amazonas e Pará que apresentam um indicador alto no que tange a cura da tuberculose nos soropositivos, um quantitativo bem expressivo. Isto deve-se a alguns fatores como: diagnóstico precoce e garantir que estes pacientes não abandonem o tratamento, são fatores decisivos e que podem levar a cura. Assim, como nos demais estados, seu percentual de cura, não é discrepante ao número de infectados.

A taxa de mortalidade no brasil sofreu uma redução, embora ainda alarmante, cerca de 6.000 óbitos são notificados todo ano, sendo sua maior incidência estados com as maiores taxas de mortalidade nos estados do Amazonas e Pará, seguido 
de Rondônia, Roraima e Tocantins mantiveram sua posição. A taxa de mortalidade de 5,5 casos por 100.000 habitantes, de acordo com o Gráfico 4, o declínio pode ser explicado por melhor atenção ao doente, pelo uso de regimes medicamentosos adequados e, é evidente, por uma melhora nas condições de vida da população brasileira.

O Estado do Amazonas possui a maior taxa de incidência da TB no Brasil, com coeficiente de 70,1 casos por 100 mil habitantes. O maior número de casos no estado concentra - se na capital Manaus, que tem um coeficiente de 98,3 casos por 100 mim habitantes, valor acima de coeficiente regional e nacional de 37,4 e 41 por 100 mil habitantes, respectivamente (Brasil, 2016).

O estado do Amazonas registrou 2.319 casos novos da doença ou 62,1/100 mil habitantes, que equivale a maior taxa de incidência de toda a região norte do país. O Brasil integra o grupo de 22 países que concentram $80 \%$ da carga da tuberculose do mundo, tendo registrado 73.778 casos novos 38.4/100 mil habitantes.

No entanto, de acordo com o Gráfico 5, foi possível observar que os números de casos por abandono de tratamento foram significativos, principalmente no AM (766), PA (537) e RO (171) por casos confirmados de abandono de tratamento. Com a finalidade de corrigir ou, pelo menos, minorar a situação epidemiológica da doença no Brasil, foi lançado o Programa Nacional de Controle da Tuberculose (PNCT), definindo a doença como questão de prioridade de saúde pública (Barbosa \& Costa, 2012).

Configura-se abandono do tratamento quando o paciente permanece faltoso por mais de 60 dias após a data da última consulta ou quando deixa de tomar o medicamento por 30 dias ou mais. O paciente que retorna ao serviço após abandono do tratamento deve ter sua doença confirmada por nova investigação diagnóstica por baciloscopia, devendo ser solicitada cultura e teste de sensibilidade (TS). Além disso, reinicia-se imediatamente o esquema básico desde o início, após coleta dos exames, até o resultado da cultura e do TS. Se apresentar resistência ao exame, deve-se encaminhar ao serviço de referência no tratamento de tuberculose, para troca do esquema.

De acordo com os levantamentos bibliográficos, num panorama geral, algumas das causas relacionada ao abandono de tratamento (Gráfico 5), ocorre quando o paciente está na reta final do tratamento, momento este, em que os sintomas estão mais leves ou inexistentes, pode o mesmo pensa estar curado.

Na relação ao grau de escolaridade e abandono do tratamento, no período estudado, destacou -se o $1^{\circ}$ Grau atingindo $72 \%$, seguidos dos registros para analfabeto com $20 \%$ e para o $2^{\circ}$ Grau com $8 \%$. Pacientes com atividade de trabalho incluindo: coletador de lixo, doméstica, militar, motorista, pedreiro, serralheiro, vendedor e vigilante 64\%. No Brasil, os percentuais dos registros de alta por abandono variam entre $4,5 \%$ e $20,3 \%$.

Segundo o Programa de controle da tuberculose, no Amazonas apenas 50\% dos doentes com tuberculose multidroga resistente é investigado de forma efetiva, fato que pode propiciar a disseminação das cepas resistentes, elevando o risco de desenvolvimento da resistência primária nos possíveis contactantes (Garrido et al., 2012). Ainda de acordo com o levantamento bibliográfico, as causas verbalizadas pelos pacientes devido ao abandono do tratamento estão diretamente ligadas aos efeitos colaterais da medicação, como náuseas, vômitos, febre, fatores como melhora do seu estado de saúde, ou por motivação da própria família, a falta do conhecimento, entre outras.

\section{Conclusão}

O presente artigo teve como objetivo abordar o perfil epidemiológico dos pacientes diagnosticados com tuberculose que são portadores de HIV através de variáveis dispostas nos relatórios de epidemiológicos e cunho público disponibilizados pelo ministério da saúde. Foi possível observar a importância da vigilância epidemiológica de tuberculose em portadores de HIV na Região Norte, no período de 2011 a 2020. 
Foi possível identificar que os estados que mais apresentaram casos de portadores de HIV com tuberculose, foram os estados do Amazonas e Pará, apontando para uma necessidade maior de políticas públicas que venham a supri as necessidades de controle e prevenção de tais patologias.

Verificou -se também que nas mesmas regiões anteriormente citadas o índice de desistência do tratamento também é elevado, contribuindo para a disseminação da patologia e aumento taxa de mortalidade. Observou - se uma relação entre os níveis mais baixos de escolaridade com a desistência do tratamento; este dado demonstra a carência de ações que venham a atender as populações com menor grau de escolaridade, assim como a classe trabalhadora que muitas vezes passam mais de 8 horas de trabalho e que tem seu acesso a atenção primária prejudicado por conta dos horários de funcionamento das instituições públicas que oferecem esse tipo de assistência.

Sugere -se estudos que avaliem a eficácia das ações de saúde nas regiões em que os índices de infecção e mortalidade são mais elevados, assim como estudos que possam produzir instrumentos para a redução da desistência do tratamento.

\section{Referências}

Aragão, J. (2011). Introdução aos estudos quantitativos utilizados em pesquisas científicas. Revistas Práxis. Ano II. N ${ }^{\circ}$ 6, Agosto 2011. http://revistas.unifoa.edu.br/index.php/praxis/article/view/566>.

Barbosa, I. L., \& Costa, I. C. C. (2012). A emergência da co-infecção tuberculose-HIV no Brasil. Revista Brasileira de Geografia Médica e da Saúde, 8(15), p. 232-244, 2012. http://www.seer.ufu.br/index.php/hygeia/article/view/17350.

Barreto, M. T. S., Santos, G. M. D., Monteiro, M. J. De S. D., Jesus, R. L. R., Barbosa, G. S., \& Oliveira, V. A. (2020). Epidemiology of tuberculosis in a northeast brazilian state. Research, Society and Development, [S. 1.], 9 (7), p. e52973643, 2020. 10.33448/rsd-v9i7.3643.

Brasil. (2016). Equipe de Vigilância das Doenças Transmissíveis. Coordenadoria Geral de Vigilância em Saúde. Secretaria Municipal de Saúde de Porto Alegre. Boletim Epidemiológico. Brasilia: Ministério da Saúde, 2016. http://lproweb.procempa.com.br/pmpa/prefpoa/cgv s/usudoc/be60_fev_16.pdf.

Brasil. (2019). O SINAN. Ministério da Saúde. 2016 e atualizado em 2019. http://portalsinan.saude.gov.br/o-sinan.

Brasil. (2021). Boletim epidemiológico. Mistério Da Saúde. Tuberculose 2021. https://www.gov.br/saude/pt-br/media/pdf/2021/marco/24/b oletimtuberculose-2021_24.03.

Campos, H. S. (2006) Etiopatogenia da tuberculose e formas clínicas. Pulmao RJ. 2006;15(1):2935.

Diedrich, C. R., Mattila, J. T., Klein, E., Janssen, C., Phuah, J., Sturgeon, T.J., Montelaro, R. C., Lin, P. L., \& Flynn, J. L. (2010). Reactivation of Latent Tuberculosis in Cynomolgus Macaques Infected with SIV Is Associated with Early Peripheral T Cell Depletion and Not Virus Load. PLoS One. 2010 Março 10; 5(3):e9611. 10.1371/journal.pone.0009611. Erratum in: PLoS One. 2015;10(4):e0124221. PMID: 20224771; PMCID: PMC2835744.

Garrido, M. S., Sékula, S. B., Souza, A. B., Ramasawmy, R., Quincó, P. L., Monte, R. L., Santos, L. O., Porcuna, T. M. P., Espinosa, F. E. M., Sacareni, V., \& Santos, M. C. (2015). Temporal Distribuition of tuberculosis in the State of Amazonas, Brazil. Revista da Sociedade Brasileira de Medicina Tropical, p. 6369, 2015. http://www.scielo.br/pdf/rsbmt/v48s1/0037-8682-rsbmt-48-s1-00063.pdf

Gutierres, L. D. S., Santos, J. L. G. D., Barbosa, S. D. F. F., Maia, A. R. C., Koerich, C., \& Gonçalves, N. (2019) Adesão aos objetivos do programa de cirurgias seguras salvam vidas: Perspectivas de enfermeiros. Revista Latino-Americana. Enfermagem 2019; 27:e3108 10.1590/1518-8345.2711.3108 www.eerp.usp.br/rlae.

Hayne, L. M. P., Santos, T. A.., \& Martins, M. M. F. (2018). Perfil Clínico-Epidemiológico De Coinfecção Por Tuberculose E Hiv No Município De Salvador, Bahia, No Período De 2008 A 2017. UCSAL. http://ri.ucsal.br:8080/jspui/bitstream/pr efix/727/1/TCCLAYLAHAYNE.pdf.

Junior, C. J. D. S., Rocha, T. J. M., \& Soares, V. D. L. (2019). Aspectos clínicos e epidemiológicos da tuberculose em pacientes com HIV/aids. 52 (3): julho/agosto /setembro. Medicina (Ribeirão Preto) [Internet]. https://doi.org/10.11606/issn.2176-7262.v52i3.p231-238

Oliveira, A. V. S. D., Alfonso, A. D., Miranda, R. S., \& Oliveira, T. R. S. Epidemiological profile of tuberculosis in the northeast of Brazil: temporary series from 2008 to 2018. Research, Society and Development, [S. 1.], 9(2), p. e108922129, 2020. 10.33448/rsd-v9i2.2129.

Oms. (2021). Mortes por tuberculose aumentam pela primeira vez em mais de uma década devido à pandemia de COVID-19. Organização Mundial De Saúde. https://www.paho.org/pt/noticias/14-10-2021-mortes-por-tuberculose-aumentam-pela-primeira-vez-em-mais-uma-decada-devido.

Resende, N. H. D. (2017). Problemas Relacionados Ao Uso De Medicamentos Em Pacientes Coinfectados Com Tuberculose E Hiv/Aids. Repositório UFMG. https://repositorio.ufmg.br/bitstream/1843/BUOSB3GGS9/1/ppgmedicamento sassistfarmaceutica_nat_liahelenaresende_dissertacaomestrado.pdf.

Robbins, S. L., Kumar, V., Abbas, A. K., Fausto, N. (2005). Patologia: Bases Patológicas das doenças. $7^{\text {a }}$ edição Rio de Janeiro: Elsevier, 2005.

Sena, I. V. D. O., Flôr, S. M. C., Júnior, F. W. D., Araújo, M. B. D., Viana, R. R., \& Almeida, P. D. (2020). Estudo clínico-epidemiológico da coinfecção TBHIV em município prioritário: análise de 10 anos. Enfermería Global. 19(4) (sep. 2020), 85-119. https://doi.org/10.6018/eglobal.414741. 
Research, Society and Development, v. 10, n. 16, e184101623906, 2021

(CC BY 4.0) | ISSN 2525-3409 | DOI: http://dx.doi.org/10.33448/rsd-v10i164.23906

Silva, K. S. M., Moura, M. E. S., Lima, M. J. V., Lino, M. R. B., \& Santos, C. N. C. Coinfecção entre portadores de HIV. R. Revista Interdisciplinar, ISSNe 2317-5079, ISSN 1983-9413, 10(3), 2017, págs. 9-20.

Smeltzer, S. C., \& Bare, B. G. (1999). Tratado de enfermagem médico-cirúrgica. Guanabara Koogan; 1999.

Souza, A. C. S. V., Albuquerque, A. C. C. D., Araújo, R. A. D., Oliveira, S. F. D., \& Carvalho, C. G. N. D. Características clínico - epidemiológicas da coinfecção por tuberculose e HIV no Estado do Piauí, Brasil. Research, Society and development. https://rsdjournal.org/index.php/rsd/article/view/7415/6696.

West, A. M. M., Azevedo, A. C. M., Leal, R. M., Pacheco, T. O., \& Mello, Y. G. (2017). Estudo Epidemiológico da Coinfecção tuberculose - HIV: um comparativo entre Sergipe e Brasil. Revista Universidade Tiradentes. https://eventos.set.edu.br/sempesq/article/view/7234. 